\title{
PV Controller Modification and its Impact on Assisting PV Penetration
}

\author{
Vishnu A. Vinaya Mohanan \\ The University of Melbourne \\ Melbourne, Victoria, Australia \\ varayamparam@student.unimelb.edu.au \\ Iven Mareels \\ IBM Research Australia \\ Melbourne, Victoria, Australia \\ imareels@au1.ibm.com
}

\author{
Robin J. Evans \\ The University of Melbourne \\ Melbourne, Victoria, Australia \\ robinje@unimelb.edu.au \\ Ramachandra Rao Kolluri \\ IBM Research Australia \\ Melbourne, Victoria, Australia \\ rkolluri@au1.ibm.com
}

\begin{abstract}
Large-scale penetration of grid-following inverters into the electricity network presents various technical challenges to grid reliability. It is well-known that the ability of a grid to maintain a stable frequency is inhibited by adding traditional grid-tied photovoltaic (PV) generators. In this work, a detailed model of a simplified grid is presented, and it is shown that the proportion of PV generation and instability are positively correlated. The main instability phenomenon is captured by a Hopf Bifurcation in the field dynamics of the synchronous generator. Such a Hopf bifurcation severely constricts the feasible operating domain of the grid and may hinder normal operation. Modifying traditional grid-tied PV controllers and its impact on grid stability is assessed through small-signal, bifurcation and transient numerical analysis. Traditional PV controllers that are modified to virtual synchronous machine (VSM) type controllers show improvement in system damping. Unlike traditional grid-tied inverters, VSM inverters participate in critical modes of the synchronous generator (SG) and augment the operational domain of the SG+VSM system significantly, more importantly, almost eliminating the need for renewable energy curtailment. A case-study approach is used to present some key results on improvements in damping ratio, feasibility domain and transient stability. Finally, a feasibility domain curve is introduced and discussed in an aim to generalize the overall stability of any such system.
\end{abstract}

\section{CCS CONCEPTS}

- Hardware $\rightarrow$ Renewable energy; Power networks; Power conversion; Smart grid.

\section{KEYWORDS}

Virtual synchronous machines, PV penetration, power system stability, bifurcation analysis.

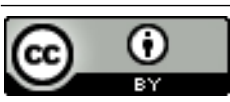

This work is licensed under a Creative Commons Attribution International 4.0 License.

e-Energy'20, fune 22-26, 2020, Virtual Event, Australia

(c) 2020 Copyright held by the owner/author(s).

ACM ISBN 978-1-4503-8009-6/20/06.

https://doi.org/10.1145/3396851.3397749
ACM Reference Format:

Vishnu A. Vinaya Mohanan, Robin J. Evans, Iven Mareels, and Ramachandra Rao Kolluri. 2020. PV Controller Modification and its Impact on Assisting PV Penetration. In The Eleventh ACM International Conference on Future Energy Systems (e-Energy'20), June 22-26, 2020, Virtual Event, Australia. ACM, New York, NY, USA, 12 pages. https://doi.org/10.1145/3396851.3397749

\section{INTRODUCTION}

Advancements in low-carbon technologies and rising energy demands are leading factors contributing to the continuous improvement of the global electricity network. The climate change threat from increased energy generation-related greenhouse gas emissions also favours the adoption of renewable energy resources (RES) [22]. To encourage the incorporation of RES into the electricity network, governments and organizations across the world are making policy amendments, providing incentives and setting short to medium term targets requiring from $15 \%$ to $30 \%$ of the total electricity generation to provided by from renewable energy resources. The target set by the European Council for 2030 is $27 \%$ [16] and the USA aims for $30 \%$ by the end of 2025 [26]. In Australia, the renewable energy target set by the Department of Environment and Energy accounts for $24 \%$ of total electricity generation [2]. Similar trends are observed in the emerging large-scale energy markets of China and India [27]. It may be argued that these targets are rather conservative, and market forces may lead to much higher penetration of renewable energy in the grid over this time horizon.

The renewable resources are predominantly asynchronous sources and require power electronic converters for interfacing them with the grid. Initially, the most intuitive way to design the controllers for the inverters is to make it follow the grid frequency as the latter is stiff parameter owing to the significant number of synchronous generators (SGs) in the network. Grid synchronisation is achieved through phase-locked loops (PLL), which enable the inverter to follow the grid frequency and latch on to it [32]. It is known that the grid-tied inverters complement the traditional grid set-up, in which the majority of the power generation is based on electromechanical sources (SG) [37]. SGs are huge rotating masses which have the inherent ability to maintain the grid frequency within the specified limits during normal as well as abnormal operations. Moreover, the matured controllers of SG tolerate the intermittent nature of PVs and wind, manage power imbalances, and stabilise the grid. However, the rising level of static (non-inertial) sources 
displaces SGs from the network, and this disrupts the operational equilibrium [19]. Removal of SGs from the system results in the reduction of overall inertia thereby causing various stability issues in the network [24]. While uptake trends show that the penetration level is likely to increase, we believe there exists a threshold above which the disintegration of SG results in unwanted interaction between the grid-following static sources and the conventional electro-mechanical sources.

\subsection{Literature review}

Detailed stability analysis of a low inertia network having a significant amount of PV (SG+PV, Fig. 3) is carried out in [31] where various stability issues were observed in the network as the noninertial generation penetration percentage is increased. Critical among them is the degradation of the system frequency response due to the displacement to synchronous generators with the PV sources and a Hopf bifurcation instability in the synchronous generator eigenmodes due to the undesirable interaction of PV controllers with the grid. These issues are pronounced when the penetration exceeds a certain threshold level which, in theory, limits the maximum amount of renewable sources that can be integrated into the grid. Bifurcations occur when the eigenvalues of the system closely associated with the field dynamics of the synchronous machine navigate beyond the imaginary axis as the PV penetration level in the network rises beyond a certain level. It is observed that the operating conditions, such as the load power factor and behavior of PV significantly influence stability. It is also noticed that the damping ratio of the eigenmode/s associated with the rotor speed of the SG declines when the non-inertial sources replace the former. In this, it can be said that the overall system stability is compromised with the large-scale integration of non-inertial sources.

The penetration level could be extended by making use of decommitted SGs as synchronous condensers (SCs) [30]. It is observed that the incorporation of SCs in the network extends the upper bound of the maximum possible penetration of renewable energy systems (RES) into the network. SCs participate in the critical modes positively by pushing them better off from the imaginary axis. It is observed that the damping ratio of rotor dynamics and field flux modes improved substantially with the incorporation of SC in the network. The capability of SC to improve the short circuit level of the system and its inherent inertia accounts for these improvements [15, 23].

Stability issues can also be addressed by modifying the grid-tied inverter controllers. Several controller modifications that suit the evolving network needs have been proposed in recent literature. The most well-known among them are virtual synchronous machine (VSM) type control [6, 9, 14] and its variations [35-37]. In most of these variations, inverter controls are made to emulate synchronous machines [8]. The advantage of modifying conventional grid-following systems to virtual synchronous machines is that the grid sees it a natural inclusion and its synchronization is through power interactions rather than a PLL $[18,37]$. Moreover, VSM also consists of parameters such as damping coefficient, inertia and virtual winding parameters (resistance, inductance) that are programmable for optimum response.
Virtual machines can be classified based on their control architecture $[10,13]$, and each configuration have certain advantages and disadvantages. Based on the reference signal fed from the virtual synchronous machine model to the inverter controller, VSMs are classified into current reference model [9], voltage reference model [37], cascade control [14] and power reference model. Details about these classifications are provided in [13]. To reiterate, although these configurations have different control architectures, the underlying principal of all these versions are the emulation of SG inertia and the droop control mechanism to control the voltage and power flow.

\subsection{Contribution and organisation}

In this paper, network augmentation through modification of PV inverter controllers is analysed from a transient and small-signal stability perspective. However, unlike most works in the past - this problem is approached using bifurcation analysis. The small-signal analysis shows that the SG+VSM system offers significant advantages over an SG+PV system when compared against increasing $\mathrm{PV}$ penetration. A key finding is that the Hopf bifurcation in the synchronous generator's field-flux mode disappears through such an augmentation. The nominal bifurcation point is extended and an increase in maximum possible PV penetration is observed. Transient response of SG+VSM system demonstrates improvements in both frequency and voltage profile of the modelled network.

It is shown that the PV penetration level in the modified system is only limited by the classical voltage stability issues which could be mitigated to some extent with the aid of VAR compensators. To verify this, SG+VSM system is augmented with an SC at the load bus. It is shown that the feasible domain, as well as the transient stability of the SG+VSM+SC system, is far ahead of other modelled configurations. Finally, a bifurcation analysis based operating domain is identified which is capable of outlining system stability in terms of PV penetration, SG contribution and loading.

The rest of the paper is organized as follows. Details of the mathematical modelling of the VSM system and other system components are provided in Section-2. Stability analysis of SG+VSM system is carried out in Section-3. The stability margins of SG+PV system is compared with SG+VSM system in this section. The impact of the synchronous condenser on the stability of SG+VSM system is assessed in Section-4. Finally, the concluding remarks are provided in Section-5. All the key assumptions made in this work and their relevance is described in Appendix A.1.

\section{SYSTEM MATHEMATICAL MODEL}

\subsection{Modeling of the VSM system}

Virtual synchronous machine model considered in this analysis is based on models proposed in [11, 14, 18, 21]. We have modified these models by implementing an additional virtual machine stabilizer (VMS) to enhance the transient response of the system. The modified system is shown in Fig. 1. This control diagram details the emulation of the mechanical and electrical side of the synchronous machine, virtual stator windings, and also the inner-loop current controllers of the inverter. Mathematical descriptions of each of the sub-modules are explained in the subsequent sections. 


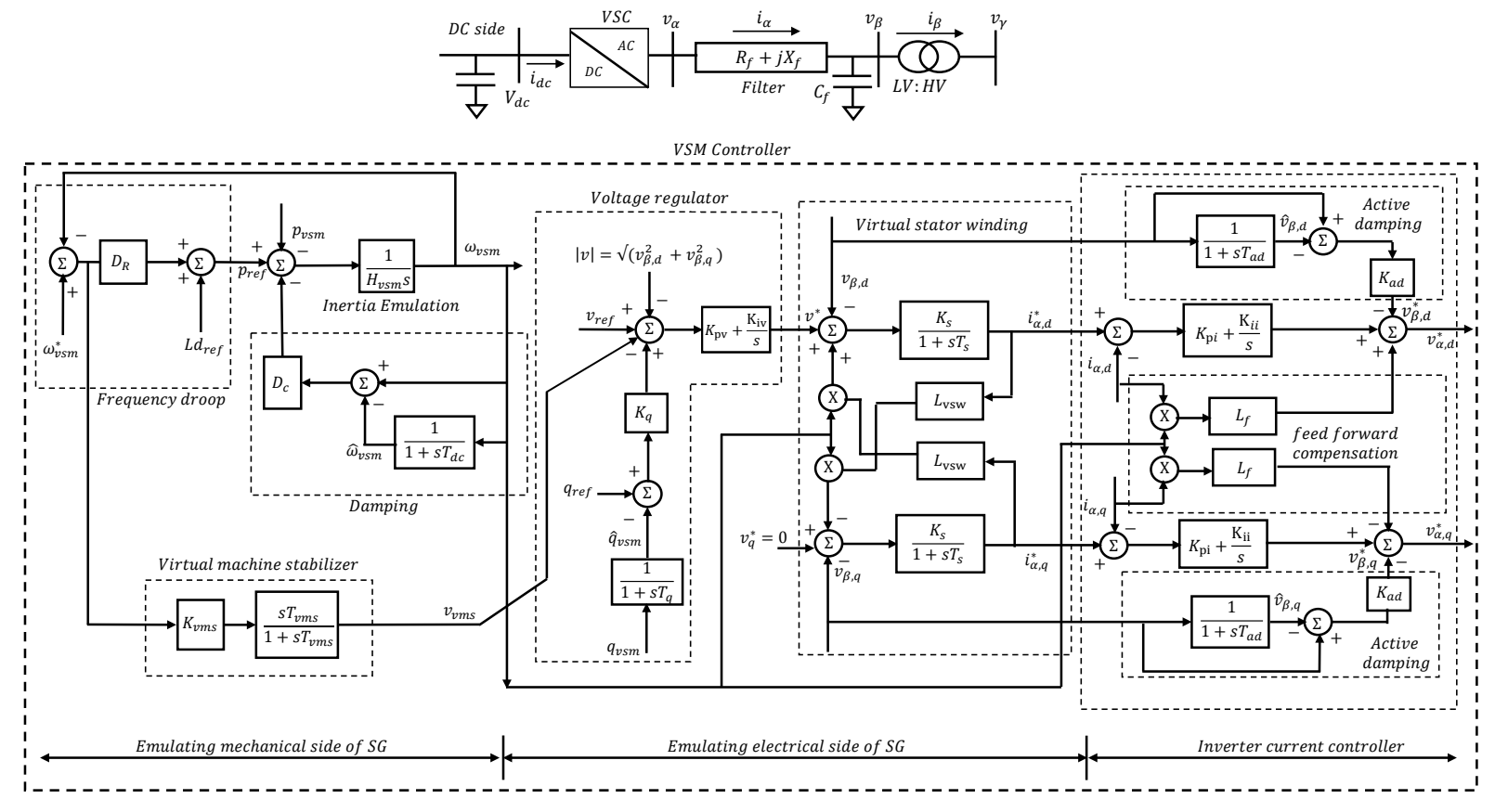

Figure 1: Control diagram of the VSM system

\subsection{Controller side of the VSM}

The control diagram of a VSM system is shown in Fig. 1. VSM controller has essentially three basic sections: emulation of the mechanical side of SG, emulation of the electrical side of SG and the conventional current controllers of the inverter [33]. Virtual machine model adopted here is generating a current reference to the inner-loop current controllers of the inverter.

2.2.1 Emulation of the mechanical side of SG. In Fig. 1, frequency droop, damping and inertia emulation altogether constitutes the mechanical side of VSM.

Frequency droop controller in SG system varies its output power based on the deviation of SG frequency from a standard frequency. Droop mechanism is essential for the uniform power-sharing between multiple sources [7]. In SG, input power is varied by adjusting control valves to regulate steam input to achieve the power balance.

In VSM, droop mechanism is implemented using similar logic as that of an SG. Virtual angular frequency of VSM is compared with a reference value and the difference is amplified and added with load reference point to achieve a steady equilibrium. The droop coefficients can be calculated as $\frac{\Delta P}{\Delta \omega}$. It is common to allow a $5 \%$ change in frequency change in return of a $100 \%$ change in power output. Similar droop regulation is provided for the VSM in this current paper as well. Dynamics of the frequency droop controller of the VSM can be represented as

$$
P_{\text {ref }}=\left(\omega_{v s m}^{*}-\omega_{v s m}\right) D_{R}+L d_{r e f},
$$

where $\omega_{v s m}^{*}$ is the reference frequency, $D_{R}$ is the droop coefficient, $L d_{r e f}$ is the load reference point. In this analysis, we have not considered an automatic generation controller and hence fixed load reference point at a constant.
Virtual damping is introduced into the VSM model-based on [21] and it can be represented as

$$
\frac{d \hat{\omega}_{v s m}}{d t}=\frac{1}{T_{d c}}\left(\omega_{v s m}-\hat{\omega}_{v s m}\right)
$$

where $T_{d c}$ is the time constant of the high-pass filter introduced to emulate the damping effect. Inertia emulation is based on the swing equation of conventional electro-mechanical systems [17]. From the control diagram (inertia emulation section), the change in the virtual angular frequency of the VSM system is represented as:

$$
\frac{d \omega_{v s m}}{d t}=\frac{1}{H_{v s m}}\left(P_{r e f}-P_{v s m}-D_{c}\left(\omega_{v s m}-\hat{\omega}_{v s m}\right)\right),
$$

where $H_{v s m}, D_{c}$ are the virtual inertia and virtual damping coefficient respectively, $P_{v s m}$ is the active power output of VSM which can be calculated as $P_{v s m}=v_{\beta, d} i_{\beta, d}+v_{\beta, q} i_{\beta, q}$.

2.2.2 Emulation of the electrical side of SG. Electrical side of VSM system mainly constitutes of voltage regulator and virtual stator windings. Voltage regulator regulates the input voltage to virtual stator winding using a proportional integral (PI) controller. It is a droop-like mechanism to control the terminal voltage based on ratio of voltage deviation with reactive power deviation [21, 37]. Mathematical representation of the voltage regulator is as follows:

$$
\frac{d \hat{q}_{v s m}}{d t}=\frac{1}{T_{q}}\left(q_{v s m}-\hat{q}_{v s m}\right)
$$

where $q_{v s m}$ is the reactive power output of the VSM given by $q_{v s m}=\left(v_{\beta, q} i_{\beta, d}-v_{\beta, d} i_{\beta, q}\right)$ and $T_{q}$ is the measurement filter time constant. 

as

The output voltage signal from the PI controller can be obtained

$$
v^{*}=\left(K_{p v}+K_{i v} \int\right)\left(v_{r e f}-|v|-v_{v m s}+k_{q}\left(q_{r e f}-\hat{q}_{v s m}\right)\right.
$$

where $v_{r e f}$ is the reference voltage, $|v|$ is the bus voltage magnitude, $v_{v m s}$ is the stabilizing signal from virtual machine stabilizer, $K_{p v}, K_{i v}$ are the PI controller gains and $k_{q}$ is the reactive-droop coefficient. In equation (5), the integral dynamics of the PI controller can be formulated as follows.

$$
\frac{d \Phi_{1}}{d t}=k_{i v}\left(v_{r e f}-|v|-v_{v m s}+k_{q}\left(q_{r e f}-\hat{q}_{v s m}\right)\right),
$$

where $\Phi_{1}$ is the state variable representing the integral term in the PI controller. The final induced voltage can be represented as,

$$
v^{*}=k_{p v}\left(v_{r e f}-|v|-v_{v m s}+k_{q}\left(q_{r e f}-\hat{q}_{v s m}\right)\right)+\Phi_{1}
$$

A virtual stator winding is modelled to emulate the behavior of the virtual machine. The output of the voltage regulator represents the induced voltage of virtual SG model. The output current from the virtual machine model is fed as the reference current to the inner-loop current controller of the inverter. The output current from the virtual stator winding (refer virtual stator winding section in Fig. 1) is obtained as

$$
\begin{aligned}
& \frac{d i_{v s w, d}}{d t}=\frac{1}{T_{s}}\left(K_{s}\left(v^{*}-v_{\beta, d}+\omega_{v s m} L_{s} i_{v s w, q}\right)-i_{v s w, d}\right), \\
& \frac{d i_{v s w, q}}{d t}=\frac{1}{T_{s}}\left(K_{s}\left(0-v_{\beta, q}-\omega_{v s m} L_{s} i_{v s w, d}\right)-i_{v s w, q}\right),
\end{aligned}
$$

where $K_{s}=\frac{1}{R_{s}}, T_{s}=\frac{L_{s}}{R_{s}}$. Here, $R_{s}, L_{s}$ are the virtual stator winding resistance and inductance, respectively.

An additional damping signal is introduced to improve the transient response of the VSM system. A new controller called virtual machine stabilizer (VMS) generates an additional signal $V_{v m s}$ in response to the frequency deviation. Deviation in VSM frequency $\omega_{\text {vsm }}$ from the nominal value is amplified and fed to the voltage controller through a washout block - a function that prevents the action of the stabilizer during steady (or slow load following) change in frequency.

At steady state, the $\omega_{v s m}^{*}$ stabilizes to $\omega_{v s m}$ and hence the output of the stabilizer becomes zero. During transients, the washout block allows the signal to pass through. The dynamics of VSM can be represented as follows: The input signal to the washout block is

$$
\omega_{v s m}^{*}-\omega_{v s m} \text {. }
$$

The washout block can be split into two first-order blocks, $\left(\frac{1}{1+s T_{v m s}}\right)$, $\left(s T_{v m s}\right)$. Let the output of the first block is $\hat{V}_{v m s}$. Then,

$$
\frac{d \hat{V}_{v m s}}{d t}=\frac{1}{T_{v m s}}\left(K_{v m s}\left(\omega_{v s m}^{*}-\omega_{v s m}\right)-\hat{V}_{v m s}\right) .
$$

The output from the second block can be obtained as

$$
V_{v m s}=T_{v m s} \frac{d \hat{V}_{v m s}}{d t} .
$$

Substituting (11) in (12) gives

$$
V_{v m s}=K_{v m s}\left(\omega_{v s m}^{*}-\omega_{v s m}\right)-\hat{V}_{v m s},
$$

where $T_{v m s}, K_{v m s}$ are the washout time constant and gain respectively.
Current controller is a typical PI controller used in the traditional grid-tied inverter system [33, 34]. Current controller modulates the output voltage of the inverter to track the reference current. Additional feed-forward signal is provided to avoid undesirable start-up transients. Feed-forward harmonic damping filter helps to eliminate switching harmonics [21,34]. Dynamics of the current controller can be represented as follows:

The mathematical representation of the PI controller is

$$
\left(K_{p i}+K_{i i} \int\right)\left(i_{v s w, d q}-i_{\alpha, d q}\right)
$$

In equation (14), the state variable representing the integral term of the PI controller is written as follows

$$
\begin{aligned}
& \frac{d \phi_{2}}{d t}=K_{i i}\left(i_{v s w, d}-i_{\alpha, d}\right), \\
& \frac{d \phi_{3}}{d t}=K_{i i}\left(i_{v s w, q}-i_{\alpha, q}\right) .
\end{aligned}
$$

The dynamics of the feed-forward filter are represented as

$$
\begin{gathered}
\frac{d \hat{v}_{\beta, d}}{d t}=\frac{1}{T_{f d}}\left(v_{\beta, d}-\hat{v}_{\beta, d}\right), \\
\frac{d \hat{v}_{\beta, q}}{d t}=\frac{1}{T_{f d}}\left(v_{\beta, q}-\hat{v}_{\beta, q}\right) . \\
v_{\beta, d}^{*}=K_{f d}\left(v_{\beta, d}-\hat{v}_{\beta, d}\right), \\
v_{\beta, q}^{*}=K_{f d}\left(v_{\beta, q}-\hat{v}_{\beta, q}\right) .
\end{gathered}
$$

The controlled output voltage of the VSM is obtained as

$$
\begin{aligned}
& v_{\alpha, d}=k_{p i}\left(i_{v s w, d}-i_{\alpha, d}\right)+\phi_{2}-\omega_{v s m} L_{f} i_{\alpha, q}-v_{\beta, d}^{*}, \\
& v_{\alpha, q}=k_{p i}\left(i_{v s w, q}-i_{\alpha, q}\right)+\phi_{3}+\omega_{v s m} L_{f} i_{\alpha, d}-v_{\beta, q}^{*} .
\end{aligned}
$$

2.2.3 AC side of the grid connected VSM. For simplifying the analysis, modeling of VSM system is carried out in $d-q$ reference frame [25]. The AC side dynamics of the VSM system are formulated based on the filter inductance current and the filter capacitor voltage. From Fig. 1, the filter inductor current in $d-q$ can be represented as

$$
\begin{aligned}
& \frac{d i_{\alpha, d}}{d t}=\frac{1}{L_{f}}\left(v_{\alpha, d}-v_{\beta, d}-R_{f} i_{\alpha, d}+\omega_{v s m} L_{f} i_{\alpha, q}\right), \\
& \frac{d i_{\alpha, q}}{d t}=\frac{1}{L_{f}}\left(v_{\alpha, q}-v_{\beta, q}-R_{f} i_{\alpha, q}-\omega_{v s m} L_{f} i_{\alpha, d}\right),
\end{aligned}
$$

where $L_{f}, R_{f}$ is the filter inductance and resistance respectively. Similarly, dynamic equation for filter capacitor voltage in $d-q$ frame is

$$
\begin{aligned}
& \frac{d v_{\beta, d}}{d t}=\frac{1}{C_{f}}\left(i_{\alpha, d}-i_{\beta, d}+\omega_{v s m} C_{f} v_{\beta, q}\right), \\
& \frac{d v_{\beta, q}}{d t}=\frac{1}{C_{f}}\left(i_{\alpha, q}-i_{\beta, q}-\omega_{v s m} C_{f} v_{\beta, d}\right),
\end{aligned}
$$

where $C_{f}$ is the filter capacitance. The dynamics equation for the grid-side inductor current ( combined inductance of transformer and transmission line) in $d-q$ frame is

$$
\begin{aligned}
& \frac{d i_{\beta, d}}{d t}=\frac{1}{L_{t}}\left(v_{\beta, d}-v_{\gamma, d}-R_{t} i_{\beta, d}+\omega_{v s m} L_{t} i_{\beta, q}\right), \\
& \frac{d i_{\beta, q}}{d t}=\frac{1}{L_{t}}\left(v_{\beta, q}-v_{\gamma, q}-R_{t} i_{\beta, q}-\omega_{v s m} L_{t} i_{\beta, d}\right),
\end{aligned}
$$


where $L_{t}, R_{t}$ is the combined transformer and transmission line inductance and resistance respectively. Here VSM system interconnection does not require a PLL because it emulates a synchronous machine and its dynamics are intertwined with the network dynamics - specially in terms of phase angle and frequency [21]. However, to interconnect the VSM system to a grid, the interacting variables need to be transformed in to the suitable reference frame ( $v_{\gamma}$ in this case).

The transformation can be done using the matrix

$$
\left[\begin{array}{l}
f_{d} \\
f_{q}
\end{array}\right]=\left[\begin{array}{cc}
\cos (\delta) & \sin (\delta) \\
-\sin (\delta) & \cos (\delta)
\end{array}\right]\left[\begin{array}{c}
F_{D} \\
F_{Q}
\end{array}\right],
$$

where, $\left[f_{d} ; f_{q}\right]$ represents variables in local VSM reference frame, $\left[F_{D} ; F_{Q}\right]$ represents the variables in global reference frame and $\delta$, the new state variable is

$$
\frac{d \delta}{d t}=\omega_{v s m}-\omega_{g r i d}
$$

where $\omega_{\text {grid }}$ is the grid frequency. Equation (1-30) represents the combined VSM system connected to the grid.

In this analysis, we have assumed that the VSM is equipped with a battery storage system at the DC side to provide inertial and governor response during frequency deviation events. It should be noted that a VSM operating at its maximum rated capacity and not having a battery storage cannot provide any frequency support to the grid. Recent advancements in battery technologies along with the reduction in costs revealed that batteries are being considered for inertial support all over the world [12]. In Australia, multiple battery energy storage systems (BESS), for instance, $30 \mathrm{MW}$ Ballarat BESS, $25 \mathrm{MW}$ Gannawarra BESS are already commissioned for frequency ancillary services [5]. Hence, we consider it a reasonable approach to model VSM with battery energy storage for frequency support.

\subsection{Synchronous generator and controllers}

SG is represented as an $8^{\text {th }}$ order model, which considers the dynamics of 3 damper windings ( 1 in $d$-axis and 2 in $q$-axis). A simplified version of the standard IEEE type ST1C excitation system model is used to represent the excitation system of the SG [3]. Simplified version of IEEE Type PSS1A power system stabilizer (PSS) model is considered in this analysis. The steam turbine is represented as a non-reheat turbine and the governor is represented as an electrohydraulic speed governor with steam feedback [1, 28].

\subsection{Synchronous Condenser}

Synchronous condenser considered in the analysis has a similar rating as that of the synchronous generator. Modelling of the SC is identical to that of an SG albeit the changes to its mechanical part and associated controllers. State variables of the SC have similar name and meaning as of those in the SG model. However, to avoid confusion a subscript ' $s c$ ' is added to these variables. The SC is decoupled from the prime movers $\left(T_{m s c}=0\right)$ and the inertia constant $\left(H_{s c}\right)$ is reduced to 1 p.u. to account for the reduction in turbine inertia [20]. Unlike the SG which has a governor system to control the input mechanical power, there is no governor in the SC - as it supports no mechanical load. As opposed to the excitation system used for the SG, we have used a first-order model to represent the

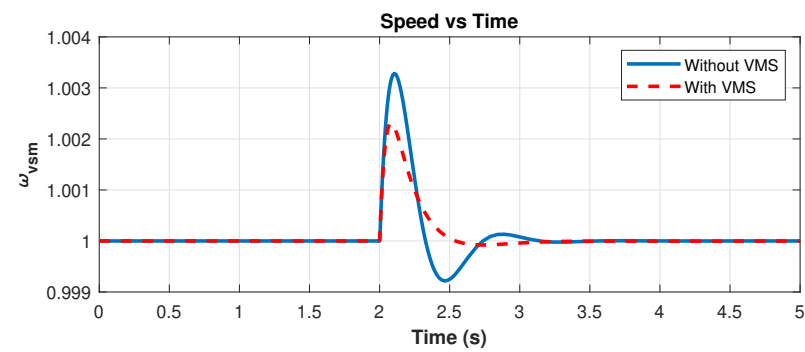

Figure 2: Evolution of the VSM angular velocity in pu.

automatic voltage regulator (AVR) for the SC for simplifying the analysis.

\subsection{Transmission line, load and PV penetration}

Transmission lines are modelled based on the dynamics of the shunt capacitance and the line inductance. The load is modelled as a constant power load and the load current in the synchronous frame is calculated from the $d-q$ component of the bus voltage $v_{2}$ and the active and reactive component of the load $\left(P_{L}, Q_{L}\right)$. The PV penetration factor is defined as:

$$
P V_{p n}=\frac{\text { Total PV generation }(\mathrm{MW})}{\text { Total generation }(\mathrm{MW})}
$$

To elaborate, a PV penetration factor of 1 represents a system that is fully supplied by PV generation.

\subsection{Performance evaluation of the virtual machine stabilizer}

This section analyses the performance of the virtual machine stabilizer introduced in this work. Transient stability analysis of a grid-connected VSM system with and without the virtual machine stabilizer is shown in Fig. 2. It is shown that the evolution of the angular velocity of a grid-connected VSM system with a virtual machine stabilizer significantly is better. The virtual machine stabilizer limits the overshoot and provides improved damping. Such a behavior is a resultant of the damping effect of the VMS on transient $v^{*}$. To illustrate this, let us consider a sudden rise in load causing a frequency dip, this will, in turn, cause a surge increase in the VSM output resulting in large frequency oscillations. However, with the incorporation of VMS stabiliser, a sudden rise in load makes $\omega_{v s m}<\omega_{v s m}^{*}$, which makes $V_{v m s}$ increasingly positive. Hence the change in $v^{*}$ will be lesser compared to a system not having a stabiliser. This improves the damping and controls the overshoot.

To elaborate the stability improvement from VMS, a small-signal analysis of the grid-connected VSM system with and without virtual machine stabilizer is performed. Both systems are evaluated at identical operating conditions. The virtual rotor speed, $\omega_{v s m}$ of the VSM has drifted away from the imaginary axis from $\lambda_{1,2}=$ $-8.27 \pm 4.39 i$ to $\lambda_{1,2}=-35.4 \pm 6.15 i$ with the inclusion of the VMS shown a significant improvement in the stability margin. The damping of this corresponding mode has improved from 0.88 to 0.99 , an $11 \%$ rise in damping. In the modified system, it is observed 


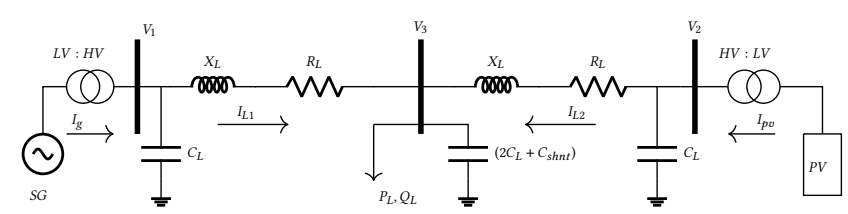

Figure 3: Single line diagram of SG-PV system.

Table 1: Dominant eigenvalues of SG-VSM and SG-PV system for $P_{L}=0.9$ p.u and $p f=0.95$ lag.

\begin{tabular}{|l|l|l|l|l|}
\hline \multicolumn{5}{|c|}{ Eigenvalue Comparison } \\
\hline EV & SG-VSM & Participation & SG-PV & Participation \\
\hline$\lambda_{1}$ & -.6 & agc, pss & -0.484 & agc, $\omega_{r}$ \\
$\lambda_{2}$ & -0.956 & agc, $\omega_{r}, \omega_{\text {vsm }}$ & -.587 & $p s s$ \\
& & $\hat{\omega}_{\text {vsm }}, p s s$ & & \\
$\lambda_{3}$ & -1 & $p s s$ & -1 & $p s s$ \\
$\lambda_{4,5}$ & $-1.34 \pm 1.51 i$ & agc, $P_{g v}, T_{m}, \omega_{r}$ & $-0.547 \pm 2.25 i$ & $P_{g v}, T_{m}, \omega_{r}$ \\
& & $\omega_{v s m}, \hat{\omega}_{\text {vsm }}$ & & \\
$\lambda_{6}$ & -1.42 & $\psi_{1 q}$ & -0.981 & $\psi_{1 q}$ \\
$\lambda_{7,8}$ & $-3.46 \pm 6.41 i$ & $\omega_{r}, \omega_{v s m}, \hat{\omega}_{v s m}, \delta$ & - & - \\
$\lambda_{9}$ & -6.01 & $P_{g v}, T_{m}, \hat{\omega}_{\text {vsm }}$ & -2.38 & $\psi_{1 q}$ \\
$\lambda_{10,11}$ & $-8.13 \pm 15.3 i$ & $\psi_{f d}, \psi_{1 d}, \Phi_{1}$ & $-3.39 \pm 20.9 i$ & $\psi_{f d}, \psi_{1 d}$ \\
& & & & \\
\hline
\end{tabular}

that the state variable associated with the VMS controller, $\omega_{\text {vsm }}$, has a significant participation in the angular speed mode. This shows that the VSM damping can be controlled by altering the stabilizer parameters, providing an additional configurable control point.

\section{STABILITY INSIGHTS}

In this section, the performance of the system supplemented with a VSM unit is compared to one with a conventional inverter (shown in Fig. 3) in small-signal and transient stability terms. We particularly analyze the changes to the stability margins.

\subsection{Comparison of eigenvalues of SG+VSM and SG+PV systems}

Table 1 shows the dominant eigenvalues of both systems. These eigenvalues correspond to an equilibrium point for $50 \% \mathrm{PV}$ penetration with SG operating at the technical minimum level ( $45 \%$ of its capacity). Dominant mechanical mode (associated with turbinegovernor and rotor dynamics) in SG+VSM system is $\lambda_{4,5}=-1.34 \pm$ $1.54 i$. It is observed that the state variables associated with the virtual mechanical part of the VSM unit contribute to this mode. For identical operating conditions, the corresponding mechanical mode in $\mathrm{SG}+\mathrm{PV}$ system $-0.547 \pm 2.25 i$, and no participation from $\mathrm{PV}$ inverter is found. The damping ratio of this particular mode has increased significantly from 0.236 to 0.66 (a $289 \%$ increase) as the PV system emulates a synchronous generator. Moreover, the modified PV system participates in the dominant mechanical modes of the SG unit - a key finding in this study. Given that the VSM system

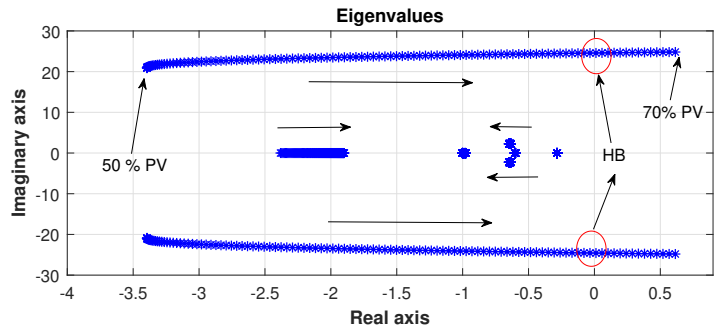

Figure 4: Dominant eigenvalues of the SG+PV system as the PV penetration varied from $50 \%$ to $70 \%$. HB: Hopf Bifurcation.

is programmable, such participation can directly be modified to achieve the desired system response.

In SG+VSM system an additional mode $\left(\lambda_{7,8}=-3.46 \pm 6.41 i\right)$ emerges, which is associated with the rotor speed of SG and VSM and virtual rotor angle $\delta$ of VSM. The damping ratio of the corresponding mode is 0.475 . Oscillatory mode associated with the field flux $\left(\psi_{f d}\right)$ of the SG in SG+PV system is found to be $\lambda_{10,11}=$ $-3.39 \pm 20.9 i$. For identical operating conditions, the corresponding mode in SG+VSM system is $-8.13 \pm 15.8 i$, which corresponds to a higher damping and consequently a higher stability margin. The damping ratio of this mode has raised from 0.16 to 0.469 , which is almost a three-fold improvement. It is observed that most dominant modes of the SG+PV system have drifted away from the imaginary axis with controller modification. The eigenvalue comparison of both systems shows that the change in the controller configuration of the PV units has a positive impact on all the critical modes of the system.

\subsection{Impact on the Hopf bifurcation}

In this section the operating domain of PV and SG in SG+VSM system is gauged and compared with that of the SG+PV system. Small-signal analysis carried out on both systems for varying levels of PV penetration. Fig. 4 shows the movement of the dominant eigenvalues of the SG+PV system when the PV penetration is varied from $50 \%$ to $70 \%$. Evidently, the field flux modes of the synchronous generator undergo a Hopf bifurcation when the PV penetration reaches around $68 \%$ of the total generation.

A similar analysis is carried out in SG+VSM system for identical operating condition and the corresponding plot is shown in Fig. 5. It is observed that the real part of critical mode associated with the field flux of SG has drifted from -8.21 to -7.742 as the PV penetration is varied from $50-80 \%$. Even with $80 \%$ of PV penetration, all the dominant modes of the system are away from imaginary axis, leaving enough margin to delay Hopf bifurcation. This shows that the change in the controller configuration has clearly improved the system stability by postponing the Hopf Bifurcation instability. 
PV Controller Modification and its Impact on Assisting PV Penetration

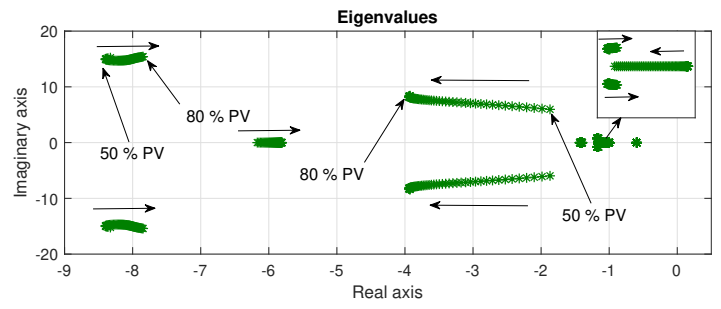

Figure 5: Dominant eigenvalues of the SG+VSM system as the PV penetration varied from $50 \%$ to $80 \%$. Bifurcation.

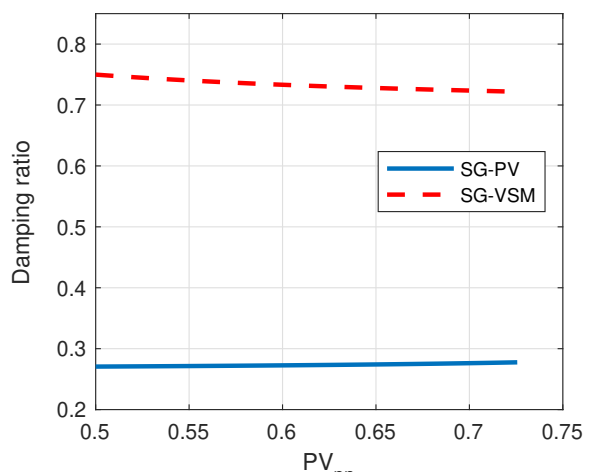

Figure 6: Loci of the damping ratio of mechanical mode (turbine-governor and rotor speed) with the variation in PV penetration: Damping ratio vs $P V_{p n}$

\subsection{Impact on the damping ratio of dominant modes}

Deviation in damping ratio of the critical modes of SG+PV and SG+VSM system with the rise in the PV penetration is compared here. Fig. 6 shows the loci of the damping ratio of the mechanical mode associated with the turbine-governor and rotor speed of the SG ( $\lambda_{4,5}$ in Table 1$)$. It is observed that the damping in SG+PV is very low and it remains almost constant at 0.28 with the rise in $P V_{p n}$. On the other hand, damping of the corresponding mode in SG+VSM system is much higher and decreased slightly from 0.75 to 0.72 with the rise in $P V_{p n}$. The active participation of VSM parameters on the dominant modes accounts for this improvement.

Fig. 7 shows the deviation in the damping ratio of the mode associated with field flux $\left(\psi_{f d}, \psi_{1 d}\right)$ of SG $\left(\lambda_{10,11}\right.$ in Table 1$)$. It is observed that the alteration of PV controllers has a pronounced effect on the damping ratio of $\psi_{f d}$ mode. VSM controllers maintained almost a steady profile for the damping ratio. It has changed from 0.476 to 0.457 . Moreover, it has eliminated the Hopf bifurcation instability in the system. In SG+PV system, it can be seen that the damping ratio becomes 0 at $P V_{p n} \approx 0.68$. A further rise in $\mathrm{PV}$ penetration makes the $\mathrm{SG}+\mathrm{PV}$ system unstable.

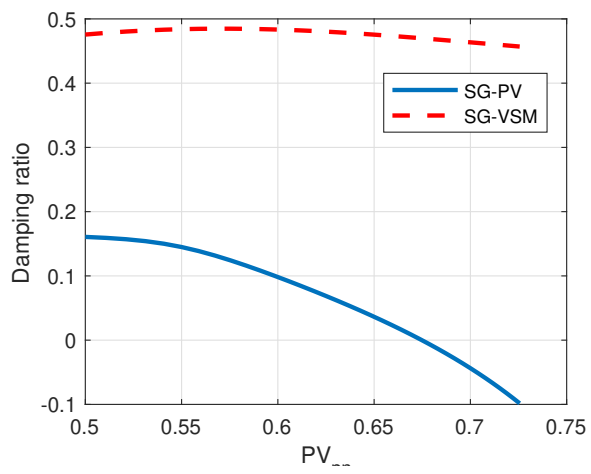

Figure 7: Loci of the damping ratio of field flux mode with the variation in PV penetration: Damping ratio vs $P V_{p n}$

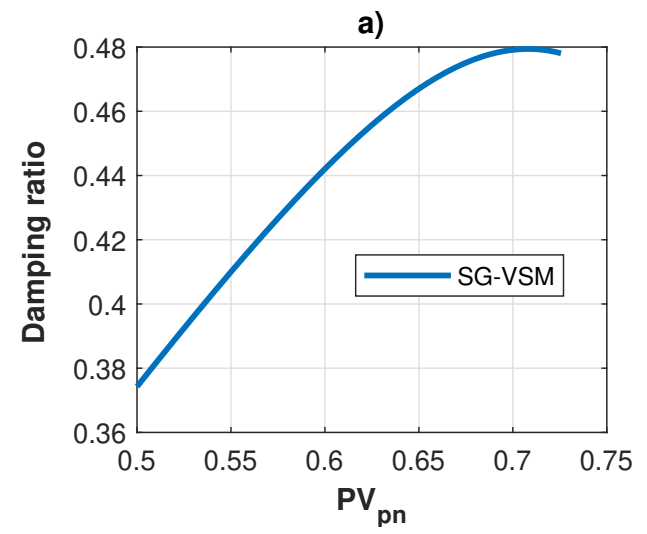

Figure 8: Loci of the damping ratio of rotor mode ( SG and VSM rotor dynamics, VSM rotor angle) with the variation in PV penetration: Damping ratio vs $P V_{p n}$

Fig. 8 shows the deviation in the damping ratio of the virtual mode, predominantly associated with $\omega_{r}, \omega_{v s m}, \hat{\omega}_{v s m}$ and $\delta$. Significant participation in this is mode found from the virtual rotor angle- $\delta$, virtual rotor speed $-\omega_{v s m}$ and SG rotor speed $\omega_{r}$. This mode is absent in the SG+PV system. It is observed that the rise in $P V_{p n}$ has positively affected the damping of this mode. Damping ratio has raised from 0.374 to 0.478 .

It can be concluded from this analysis that modification of PV controllers has in-fact have a positive effect on the damping of the critical modes. The damping ratio of all the critical modes has almost tripled as the PV system started to behave as the virtual synchronous machines.

\subsection{Impact on frequency stability}

In this section, we examine the capabilities of VSM to improve the frequency stability of the system. The impact of VSM on the frequency stability of the system is analysed by subjecting the two system topologies to a disturbance of the form of a sudden load 


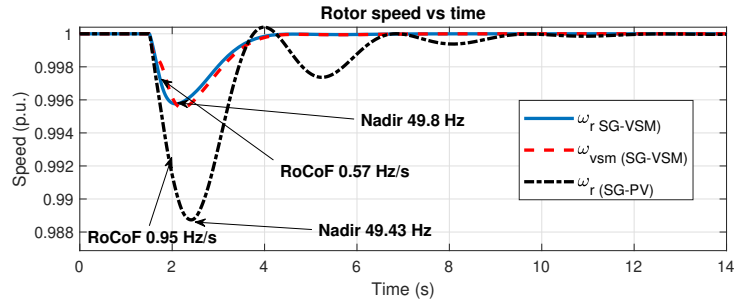

Figure 9: Evolution of SG rotor frequency in SG+PV system and SG, VSM rotor frequency in SG+VSM system.

change. At $t=1.5 \mathrm{~s}$, the active power component of the load is changed from $P_{L}=0.9$ p.u. to $P_{L}=1.05$ p.u by keeping the $\mathrm{pf}$ at 0.95 lag. The loading on the synchronous generator is kept at the technical minimum limit. Fig. 9 shows the evolution of SG and VSM rotor frequencies in two system topologies. It is identified that the frequency nadir of SG+PV system, $49.43 \mathrm{~Hz}$, is very low compared to $49.8 \mathrm{~Hz}$ of SG+VSM system. Similarly, the rate of change of frequency $(\mathrm{RoCoF})$ in $\mathrm{SG}+\mathrm{PV}$ system, $\approx 0.95 \mathrm{~Hz} / \mathrm{s}$, had improved to $\approx 0.57 \mathrm{~Hz} / \mathrm{s}$, when the PV system started to behave as a virtual synchronous machine. This shows that both frequency nadir and the RoCoF has decidedly improved SG+VSM system.

\subsection{Impact on the feasible dispatch domain}

In this section, we explicitly analyse the improvements in the combined dispatch domain of PV and SG due to the modification of PV controllers. The main aim of this analysis is to find the maximum penetration of $\mathrm{PV}$ to an existing network. The desired penetration level of PV is obtained by aggregating a large number of small VSM units. The upper-bound of maximum PV penetration is calculated by analysing all the stability limits of the system.

Fig. 10 shows the comparison of maximum penetration limit possible in two system configurations. It is observed that the upperbound in SG+VSM system is significantly larger compared to SG+PV system. Penetration level, when SG operates at technical minimum jumps from $\approx 68 \%$ to $\approx 80 \%$ when the PV controllers are modified. Hopf bifurcation stability limits no longer apply to the maximum PV penetration in the modified system. Instead, the upper-bound in SG+VSM system is set by the voltage limits of the load bus - an issue that is experienced in classical inertial systems [29].

Fig. 11 shows the combined operational domain of both system topologies. VSM controllers significantly improve the upper-bound of maximum penetration for all load power factors. The maximum penetration level is obtained when the SG operates close to its minimum level.

\subsection{Bifurcation analysis to verify the voltage stability problem}

To identify the voltage stability limit of the network, bifurcation analysis is carried out with input PV input power as the bifurcation parameter. SG is kept at the technical minimum level, and the load in the network is increased in proportion to the rise in PV generation. Fig. 12 shows the corresponding bifurcation diagram. Y-axis and

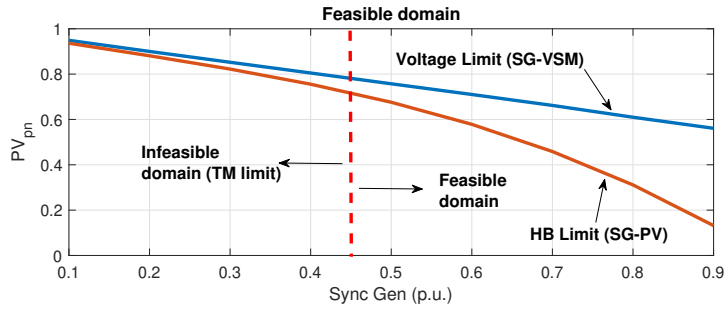

Figure 10: The dispatch limit of $\mathrm{PV}+\mathrm{SG}$ in $\mathrm{SG}+\mathrm{PV}$ and SG+VSM system for load pf=0.95 lag. $H B$ : Hopf bifurcation, $T M$ : Technical minimum.

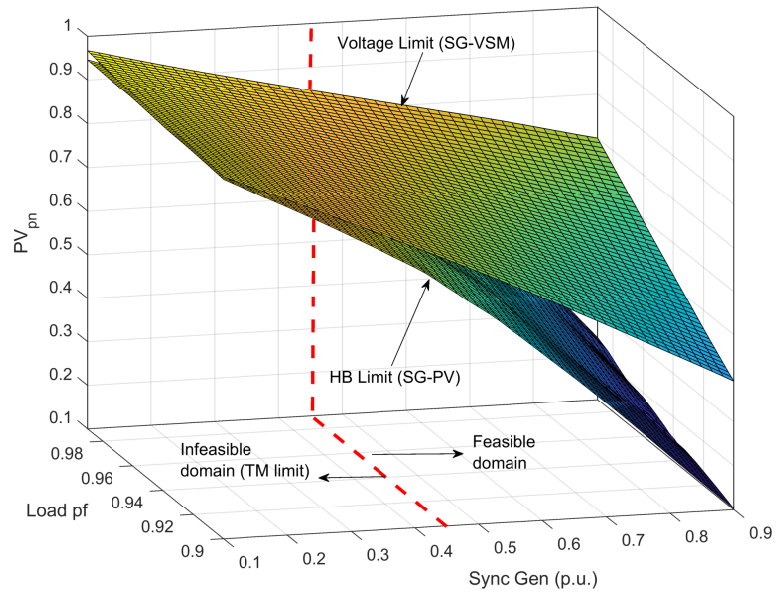

Figure 11: Comparison of the feasible dispatch domain of $\mathbf{P V + S G}$ in SG+PV and SG+VSM system. HB: Hopf bifurcation, $T M$ : Technical minimum.

$\mathrm{x}$-axis show the corresponding variation in the $d-q$ axis load bus voltage.

The bifurcation diagram is similar to the classical P-V curve [29]. The voltage magnitude of the load bus degrades and passes through into a sub-critical Hopf bifurcation at $P V_{p n}=0.88$. A further rise in $\mathrm{PV}$ power results in a neutral saddle equilibrium followed by a limit point bifurcation (saddle-node bifurcation). Reactive power shortage in the network can lead to such behavior. The system completely loses its stability when the PV penetration rises beyond the critical bifurcation value. Bifurcation point can be extended by providing adequate reactive power at the load bus, which controls the line current and line losses, thereby maintaining a nominal voltage profile.

To verify the stability of the obtained Hopf bifurcation point, we pursued plotting the extended limit cycle from the Hopf bifurcation point. Fig. 13 shows this continuation of the limit cycle from the Hopf bifurcation point, as in Fig. 12. It can be observed the the limit cycles are formed ahead of the Hopf bifurcation point and disappear at the critical Hopf bifurcation point. This shows that the Hopf bifurcation is sub-critical and there are no local stable 


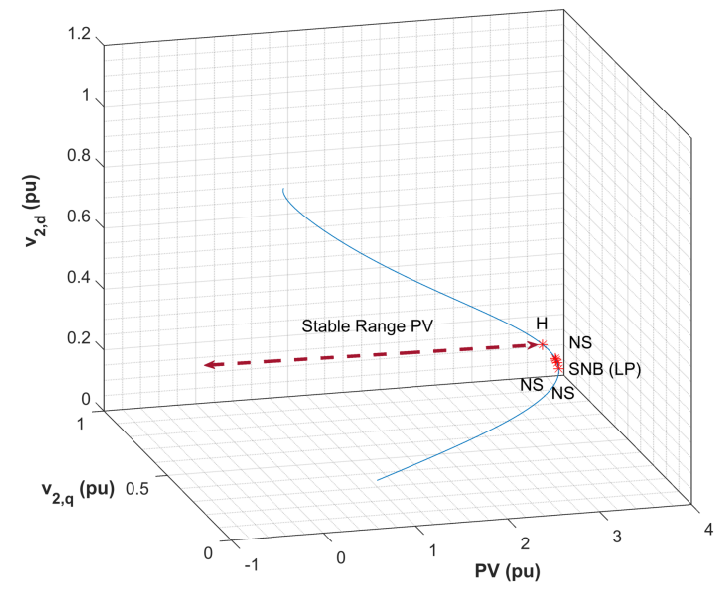

Figure 12: Bifurcation diagram with $P V$ input as the bifurcation parameter. $H$ : Hopf bifurcation $N S$ : Neutral saddle $S N B$ : Saddle node bifurcation

equilibria for the modelled system beyond the critical point. The first Lyapunov coefficient calculated at the bifurcation point is positive confirming an unstable limit cycle that bifurcates from the stable equilibrium point. This implies that the system will lose its stability due to voltage collapse as the PV penetration rises beyond this critical value.

It should be pointed out here that this stability loss due to voltage collapse can be directly attributed to the power transfer capability of the network. Electricity networks, even in a nominal SG only operation, have a limit on the maximum amount of power that can be transferred through sections of it [29]. Any modified system will remain stable for as long as the power transfer is below this limit. Network augmentation and reactive power support using static and dynamic VAR compensators including synchronous condensers can extend these limits [17].

\section{SYNCHRONOUS CONDENSER INTEGRATION AND STABILITY}

The SG+VSM system is modified by connecting SC at the load bus to potentially combat the voltage stability issues. Stability margins of the newly modified system (SG+SC+VSM) (as shown in Fig. 14) are analysed in this section. Particularly, a detailed comparison between SG+VSM system and SG+SC+VSM system is carried out to assess the expanse of the feasible dispatch domain, frequency and voltage stability.

\subsection{Transient response of SG+VSM and SG+SC+VSM system}

The transient response of SG+SC+VSM system is studied by subjecting the system to a sudden load change. At $t=1.5 \mathrm{~s}$, active power component of the load is changed from $P_{L}=0.9 \mathrm{pu}$ to $P_{L}=1.05 \mathrm{pu}$, a $15 \%$ increase in load. Fig. 15 shows the corresponding evolution of rotor frequency of SG, VSM in SG+VSM system and that of SG, SC, $\mathrm{VSM}$ in $\mathrm{SG}+\mathrm{SC}+\mathrm{VSM}$ system. SG+SC+VSM system has improved

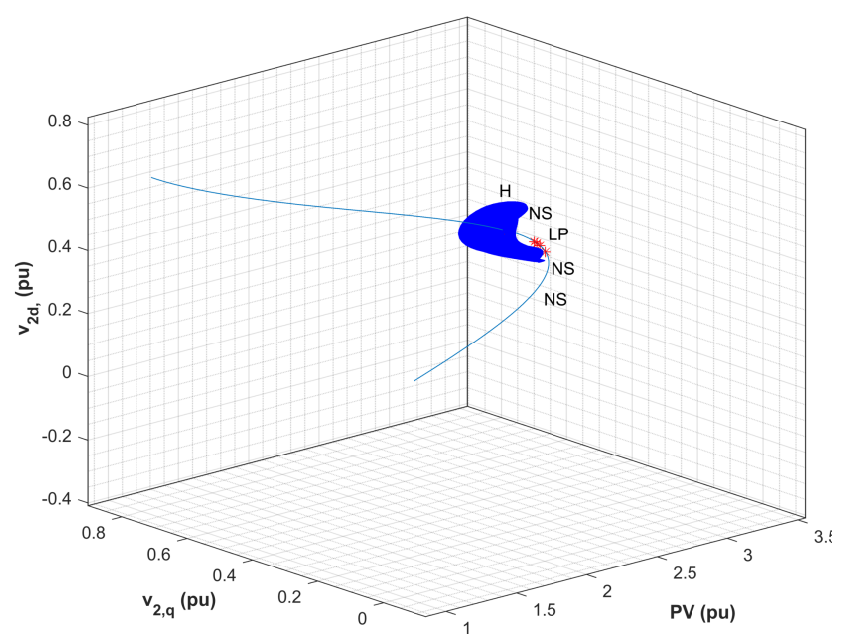

Figure 13: Continuation of the limit cycle from the Hopf bifurcation point. $H$ : Hopf bifurcation $N S$ : Neutral saddle $S N B$ : Saddle node bifurcation

frequency characteristics when compared to the SG+VSM system. The frequency nadir has raised from $49.8 \mathrm{~Hz}$ to $49.83 \mathrm{~Hz}$ and the RoCof also improved from $0.57 \mathrm{~Hz} / \mathrm{s}$ to $0.433 \mathrm{~Hz} / \mathrm{s}$.

Fig. 16 shows the corresponding evolution of bus voltage magnitudes of SG+VSM and SG+SC+VSM system. Inclusion of SC in the network has significantly improved the voltage profile of the network during transients, specifically at the SG bus $\left(v_{1}\right)$, VSM bus $\left(v_{3}\right)$ and load bus $\left(v_{2}\right)$. SC provides an adequate amount of reactive support to minimize the voltage deviation, as discussed earlier.

\subsection{Comparison of the feasible dispatch domain}

Fig. 17 shows the feasible domain of operation of PV and SG in SG+VSM and SG+SC+VSM system for a load power factor of 0.95 lag. Fig. 18 shows the complete profile of the combined operational domain of PV and SG in both systems. It is observed that for all the load power factor values, the boundary is extended with the inclusion of SC in the network.

\section{CONCLUSION}

In this paper, we analyse the stability margin of a low-inertia PV rich network when PV controllers are modified to emulate synchronous machines. It is identified that VSM type control substantially improves both small-signal and transient stability of the conventional SG+PV system. Damping of all the critical modes is improved through this modification. Moreover, the VSM type controller enables the PV system to participate in the critical mechanical modes of the SG providing additional flexibility.

The numerical analysis shows that VSM control improves the frequency stability of the system, frequency nadir is improved by $0.7 \%$, and the RoCoF is improved by $66 \%$. Adding an SC at the load bus showed that the frequency nadir and RoCoF change be improved furthermore. The inclusion of SC in the network slightly improved the frequency and voltage response of the system. This is 


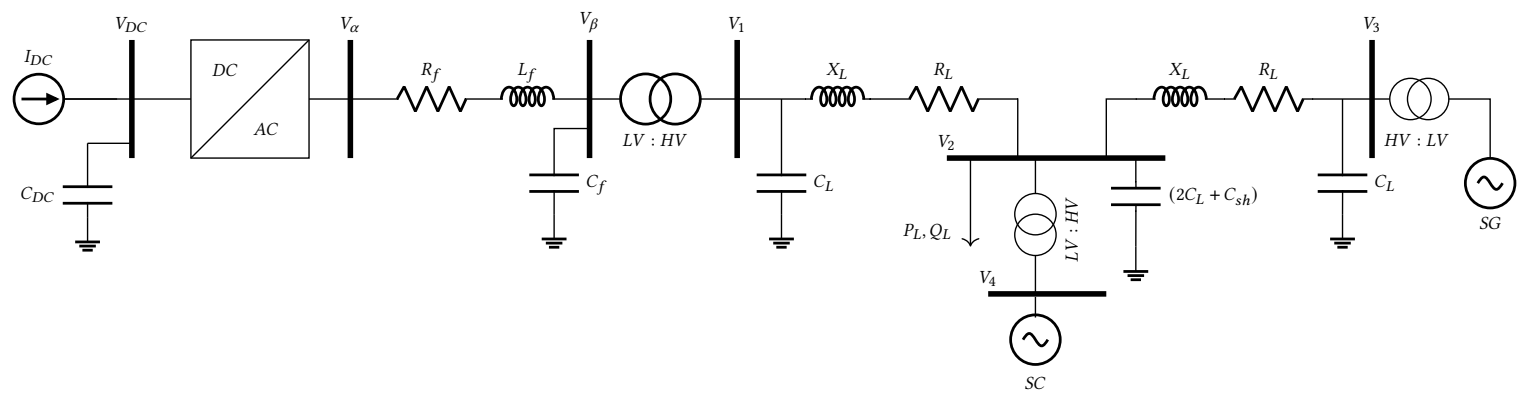

Figure 14: Single line diagram of SG-SC-VSM system

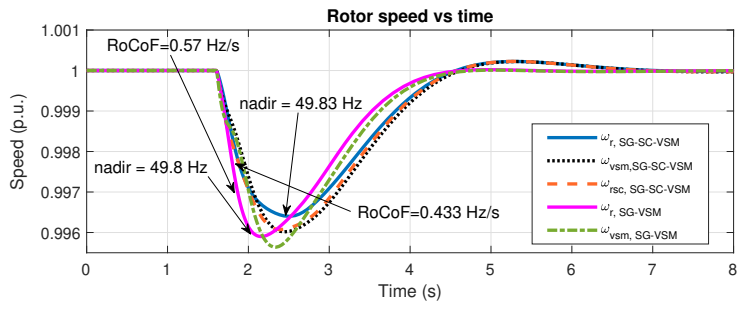

Figure 15: Evolution of SG, SC and VSM rotor frequency when SG+VSM and SG+SC+VSM systems subjected to a sudden load change
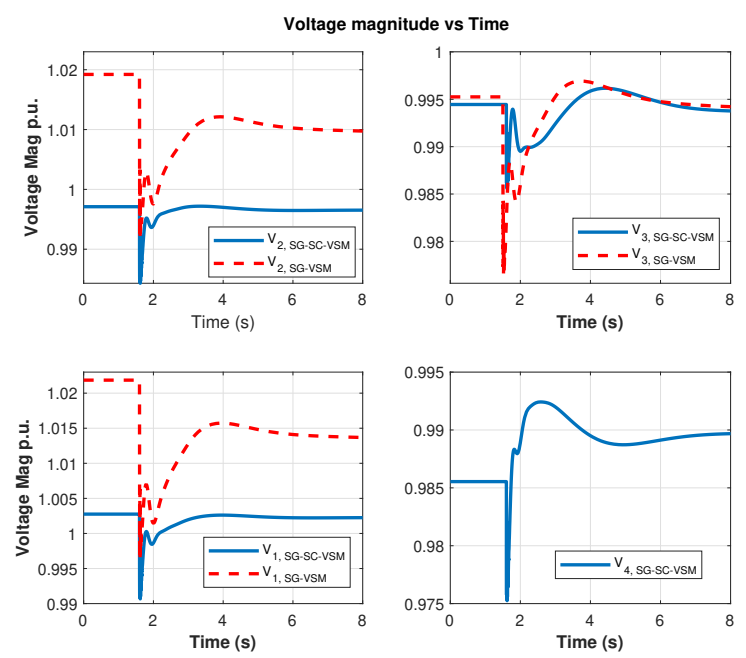

Figure 16: Evolution of voltage magnitude when SG+VSM and SG+SC+VSM systems subjected to a sudden load change

due to the additional inertia provided by the new rotating machine and its contribution to reactive power generation. The combined operational domain is also significantly improved with the inclusion of SC.

The feasibility domain curves presented in this work can be crucial in assessing the stability of large networks using a simplified

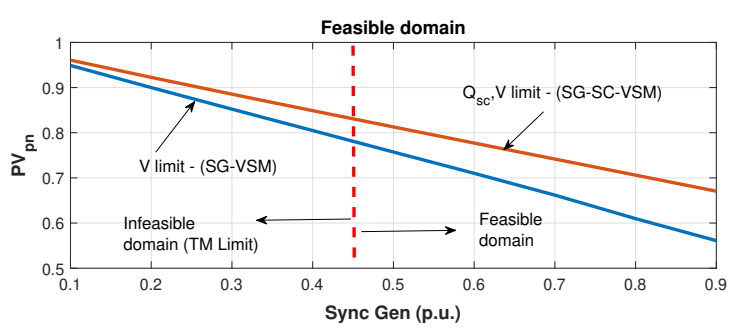

Figure 17: The dispatch limit of $\mathrm{PV}+\mathrm{SG}$ in SG+VSM and $\mathrm{SG}+\mathrm{SC}+\mathrm{VSM}$ system for $\mathrm{load} \mathbf{p f}=\mathbf{0 . 9 5} \mathrm{lag}$. $Q_{s c}: \mathrm{SC}$ reactive power, $T M$ : Technical minimum, $V$ : Voltage.

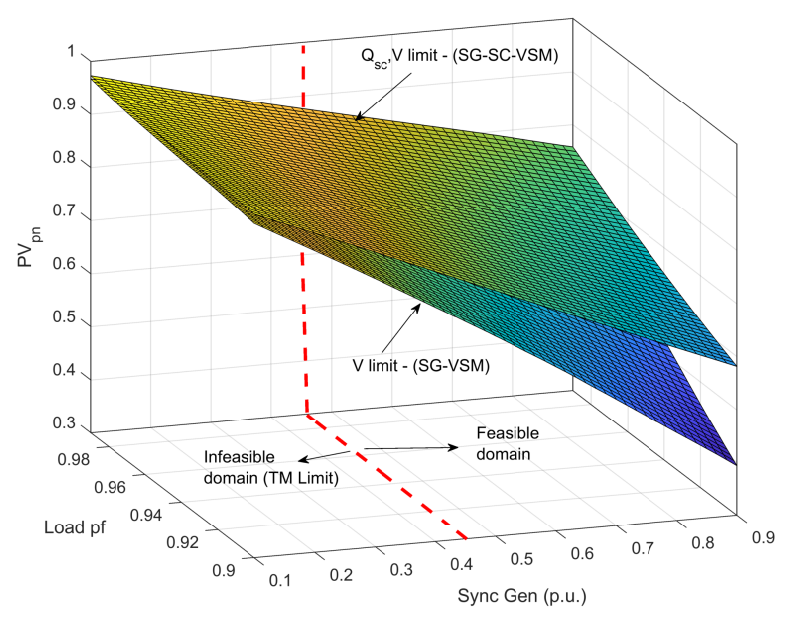

Figure 18: Comparison of the feasible dispatch domain of $\mathrm{PV}+\mathrm{SG}$ in $\mathrm{SG}+\mathrm{VSM}$ and $\mathrm{SG}+\mathrm{SC}+\mathrm{VSM}$ system. $Q_{s c}$ : $\mathrm{SC}$ reactive power, $T M$ : Technical minimum, $V$ : Voltage.

model. It can be concluded that given a bifurcation exists in the lower-order network, the behaviour persists in any higher-order system as long as there is a continuous transformation that extends the reduced-order system to its higher-order elaboration - making the dispatch domain generation a key finding in this work. Given that the used models are well-known representations, the domains 
Table 2: Parameters of a 550 MVA VSM unit

\begin{tabular}{|l|l|l|l|}
\hline \multicolumn{3}{|c|}{ Parameters } \\
\hline$L_{v s w}$ & $0.1188 \mathrm{pu}$ & $K_{i v}$ & 100 \\
$R_{v s w}$ & $0.0475 \mathrm{pu}$ & $L_{f}$ & $0.038 \mathrm{pu}$ \\
$D_{c}$ & 20 & $R_{f}$ & $0.00143 \mathrm{pu}$ \\
$D_{R}$ & 20 & $C_{f}$ & $0.16 \mathrm{pu}$ \\
$H_{v s m}$ & $3 \mathrm{~s}$ & $K_{a d}$ & 0.8 \\
$T_{d c}$ & $0.26 \mathrm{~s}$ & $T_{a d}$ & $0.02 \mathrm{~s}$ \\
$K_{p i}$ & 1.5 & $T_{v m s}$ & $2.5 \mathrm{~s}$ \\
$K_{i i}$ & 15 & $K_{v m s}$ & 50 \\
$K_{p v}$ & 0.1 & & \\
\hline
\end{tabular}

curves can be generalized to assess the stability of most similar systems.

\section{A APPENDIX}

\section{A.1 Assumptions}

We make the following simplifying assumptions

- The grid is modelled as symmetric balanced three-phase network and hence the zero sequence component in the $d-q$ frame can be ignored.

- Nonlinear elements such as saturation and hysteresis in SG dynamics are neglected.

- The SG is modelled with a sinusoidal air-gap flux distribution, to allow the use of Park's transformation with advantage.

- The PV system is modelled as a nonlinear instantaneous active power element subject to constant insolation.

- The DC and AC sides of the PV system are assumed to be decoupled, which is true from a steady-state perspective in most cases. The DC decoupling capacitor plays an important role here. This, in turn, leads to an assumption that the amount of energy available on the DC side is non-detrimental to the operation of the network/s under consideration under any presented circumstance.

- This work does not consider major topology changes or faults in its entirety.

- VSM is equipped with a battery storage system to cover the energy requirement for inertial and governor responses during frequency deviation events.

\section{B APPENDIX}

\section{B.1 Parameters considered for simulation}

Details of time constants, gains and virtual winding resistance and inductance values of the VSM unit are given in Table 2. VSM parameters considered in this analysis are derived from various literature $[11,14,18,21]$. The adopted parameters are further tuned to optimize the performance of our modified VSM.

The values of various time constants and gains of synchronous machine, AVR, PSS, turbine and governor are shown in the table 3. The parameters of the PV unit are given in Table. 4. Modelling details of these components are provided in [4, 30, 31].
Table 3: Parameters, gains and time constants used for simulation

\begin{tabular}{|l|l|l|}
\hline \multicolumn{3}{|c|}{ Parameters } \\
\hline $\begin{array}{l}\text { Synchronous } \\
\text { Generator }\end{array}$ & $\begin{array}{l}\text { Synchronous } \\
\text { Generator }\end{array}$ & Subsystem \\
\hline$R_{a}=0.003$ & $R_{f d}=0.0065$ & $K_{L F}=4.5$ \\
$R_{1 d}=0.021$ & $R_{1 q}=0.0062$ & $D R=20$ \\
$R_{2 q}=0.0234$ & $L_{a d}=1.306$ & $T_{R}=0.04$ \\
$L_{a q}=1.165$ & $L_{l}=0.15$ & $T_{G}=0.2 s$ \\
$L_{f d}=0.17$ & $L_{1 d}=0.1713$ & $T_{1}=1.65 s$ \\
$L_{1 q}=0.725$ & $L_{2 q}=0.125$ & $T_{2}=.03 s$ \\
$L_{f d}=1.31$ & $L_{11 d}=1.56$ & $T_{3}=1 s$ \\
$L_{f f d}=1.65$ & $L_{11 q}=2.08$ & $T_{4}=1 s$ \\
$L_{22 q}=1.47$ & $L_{q}=1.49$ & $K_{A}=190$ \\
$L_{d}=1.54$ & $\omega_{o}=2 \pi 50$ & $K_{S T}=10.7$ \\
\hline
\end{tabular}

Table 4: Parameters of PV system used in the analysis

\begin{tabular}{|l|l|}
\hline \multicolumn{2}{|c|}{ PV Parameters } \\
\hline$T_{a}=0.05 s$ & $T_{b}=0.02 s$ \\
$T_{c}=0.15 s$ & $T_{d}=0.05 s$ \\
$k_{p v}=18$ & $k_{i v}=5$ \\
$v e_{\max }=0.1$ & $v e_{\text {min }}=-0.1$ \\
$k p_{d c}=5$ & $k i_{d c}=50$ \\
$k p_{Q}=5$ & $k i_{Q}=50$ \\
$k p_{d}=.05$ & $k i_{d}=7.5$ \\
$k p_{q}=.05$ & $k i_{q}=7.5$ \\
\hline
\end{tabular}

\section{B.2 Per unit (p.u.) calculation}

The MVA base for per unit analysis is taken as 550 MVA and the voltage base is taken as the peak value of phase voltage at each section. The base frequency is taken as $\omega_{o}=2 \pi 50 \mathrm{~Hz}$. The synchronous generator considered in this analysis is a $550 \mathrm{MVA}, 24 \mathrm{kV}$ generator, whose parameters are provided in Table 3 . The per-unit choice for the synchronous machine stator quantities are based on the machine rating and the rotor per-unit quantities are represented based on the reciprocal per-unit system proposed in [17]. The perunit choice used for the excitation system is the non-reciprocal system which is considered as the universal choice for system studies [17]. The transmission line considered is a $500 \mathrm{kV} 100 \mathrm{~km}$ long line. The parameters of the transmission line are $R_{L}=0.025 \Omega / \mathrm{km}$, $X_{L}=0.35 \Omega / \mathrm{km}$ and $B C=5.3 \mu \mathrm{s} / \mathrm{km}$. A 125 MVAR shunt capacitor is connected at the load bus to provide reactive power support. Each PV unit considered in this analysis is of 5 MVA, $1 \mathrm{kV}$ capacity. The filter resistance and inductance have values $R_{F}=15 \mathrm{~m} \Omega, L_{F}=44 \mu$ $\mathrm{H}$ respectively. The $\mathrm{DC}$ capacitance is $C_{D C}=10 \mathrm{mF}$ and $\mathrm{DC}$ bus has a nominal voltage of $2 \mathrm{kV}$. Connection at the ac is done through a 5.5 MVA step-up transformer having an impedance of $7 \%$ on its base value. The parameter details of the PV unit is given in Table 4. Following industry practice, the PV farm is formed by aggregating all PV units together to get the desired penetration level. For this, 
we have assumed that all the PV units have identical properties. So, $n 5$ MVA PV systems are aggregated into a single PV farm of capacity $5 \times n$ MVA. The aggregated PV farm has filter resistance $\frac{R_{F}}{n} m \Omega$, inductance of $\frac{L_{F}}{n} \mu \mathrm{H}$ and DC capacitance of $n C_{D C} \mathrm{mF}$. Connection transformers are aggregated into a single transformer of rating $n \times 5.5$ MVA.

\section{REFERENCES}

[1] 1991. Dynamic models for fossil fueled steam units in power system studies IEEE Transactions on Power Systems 6, 2 (May 1991), 753-761. https://doi.org/10. $1109 / 59.76722$

[2] 2015. Renewable Energy (Electricity) Amendment Act 2015, Federal Register of Legislation,Australia. No. 90, 2015. https://www.legislation.gov.au/Details/ C2015A00090. Jun-26, 2015.

[3] 2016. IEEE Recommended Practice for Excitation System Models for Power System Stability Studies. IEEE Std 421.5-2016 (Revision of IEEE Std 421.5-2005) (Aug 2016), 1-207. https://doi.org/10.1109/IEEESTD.2016.7553421

[4] V. A. Vinaya Mohanan. 2019. The Impact of Inverter Based Systems on Power Grid Stability. Ph.D. Dissertation.

[5] Australian Renewable Energy Agency. 2019. Large-Scale Battery Storage Knowledge Sharing Report, November 2019. https://arena.gov.au/assets/2019/11/largescale-battery-storage-knowledge-sharing-report.pdf. [Accessed: 11-May-2020].

[6] H. Beck and R. Hesse. 2007. Virtual synchronous machine. In 20079 th International Conference on Electrical Power Quality and Utilisation. 1-6. https://doi.org/10. 1109/EPQU.2007.4424220

[7] A. R. Bergen and V. Vittal. 2000. Power systems analysis. Prentice Hall.

[8] K. De Brabandere, B. Bolsens, J. Van den Keybus, A. Woyte, J. Driesen, and R. Belmans. 2007. A Voltage and Frequency Droop Control Method for Paralle Inverters. IEEE Transactions on Power Electronics 22, 4 (July 2007), 1107-1115. https://doi.org/10.1109/TPEL.2007.900456

[9] Y. Chen, R. Hesse, D. Turschner, and H. Beck. 2011. Improving the grid power quality using virtual synchronous machines. In 2011 International Conference on Power Engineering, Energy and Electrical Drives. 1-6. https://doi.org/10.1109/ PowerEng.2011.6036498

[10] Y. Chen, R. Hesse, D. Turschner, and H. Beck. 2012. Comparison of methods for implementing virtual synchronous machine on inverters. In International Conference on Renewable Energies and Power Quality. 1-6.

[11] Y. Chen, R. Hesse, D. Turschner, and H. Beck. 2012. Investigation of the Virtual Synchronous Machine in the island mode. In 2012 3rd IEEE PES Innovative Smart Grid Technologies Europe (ISGT Europe). 1-6. https://doi.org/10.1109/ISGTEurope. 2012.6465648

[12] W. J Cole and A. Frazier. 2019. Cost projections for utility-scale battery storage Technical Report. National Renewable Energy Lab.(NREL), Golden, CO (United States).

[13] S. D'Arco and J. A. Suul. 2013. Virtual synchronous machines - Classification of implementations and analysis of equivalence to droop controllers for microgrids. In 2013 IEEE Grenoble Conference. 1-7. https://doi.org/10.1109/PTC.2013.6652456

[14] S. D’Arco, J. A. Suul, and O. B. Fosso. 2015. A Virtual Synchronous Machine implementation for distributed control of power converters in SmartGrids. Electric Power Systems Research 122 (2015), 180 - 197. https://doi.org/10.1016/j.epsr.2015. 01.001

[15] Y. Katsuya, Y. Mitani, and K. Tsuji. 2000. Power system stabilization by synchronous condenser with fast excitation control. In PowerCon 2000. 2000 International Conference on Power System Technology. Proceedings (Cat. No.00EX409), Vol. 3. 1563-1568 vol.3. https://doi.org/10.1109/ICPST.2000.898204

[16] B. Knopf, P. Nahmmacher, and E. Schmid. 2015. The European renewable energy target for 2030 - An impact assessment of the electricity sector. Energy Policy 85 (2015), 50 - 60. https://doi.org/10.1016/j.enpol.2015.05.010

[17] P. Kundur, N. J. Balu, and M. G. Lauby. 1994. Power system stability and control. Vol. 7. McGraw-hill New York.

[18] C. Li, R. Burgos, I. Cvetkovic, D. Boroyevich, L. Mili, and P. Rodriguez. 2014. Analysis and design of virtual synchronous machine based STATCOM controller. In 2014 IEEE 15th Workshop on Control and Modeling for Power Electronics (COMPEL). 1-6. https://doi.org/10.1109/COMPEL.2014.6877134

[19] N. W. Miller, K. Clark, and M. Shao. 2011. Frequency responsive wind plant controls: Impacts on grid performance. In 2011 IEEE Power and Energy Society General Meeting. 1-8. https://doi.org/10.1109/PES.2011.6039137

[20] N. W. Miller, M Shao, S Pajic, and R D'Aquila. 2014. Western wind and solar integration study phase 3-frequency response and transient stability. Technical Report. National Renewable Energy Lab.(NREL), Golden, CO (United States); GE Energy Management, Schenectady, NY (United States).

[21] O. Mo, S. D’Arco, and J. A. Suul. 2017. Evaluation of Virtual Synchronous Machines With Dynamic or Quasi-Stationary Machine Models. IEEE Transactions on Industrial Electronics 64, 7 (July 2017), 5952-5962. https://doi.org/10.1109/TIE 2016.2638810
[22] O. Ogunrinde, E. Shittu, and K. K. Dhanda. 2018. Investing in Renewable Energy: Reconciling Regional Policy With Renewable Energy Growth. IEEE Engineering Management Review 46, 4 (Fourthquarter 2018), 103-111. https://doi.org/10.1109/ EMR.2018.2880445

[23] J. A. Oliver, B. J. Ware, and R. C. Carruth. 1971. 345 MVA Fully Water-Cooled Synchronous Condenser for Dumont Station Part I. Application Considerations. IEEE Transactions on Power Apparatus and Systems PAS-90, 6 (Nov 1971), 27582764. https://doi.org/10.1109/TPAS.1971.292972

[24] J. O'Sullivan, A. Rogers, D. Flynn, P. Smith, A. Mullane, and M. O'Malley. 2014. Studying the Maximum Instantaneous Non-Synchronous Generation in an Island System-Frequency Stability Challenges in Ireland. IEEE Transactions on Power Systems 29, 6 (Nov 2014), 2943-2951. https://doi.org/10.1109/TPWRS.2014.2316974

[25] R. H. Park. 1929. Two-reaction theory of synchronous machines generalized method of analysis-part I. Transactions of the American Institute of Electrical Engineers 48, 3 (July 1929), 716-727. https://doi.org/10.1109/T-AIEE.1929.5055275

[26] Federal Energy Management Program. March-25, 2015. Executive Order 13693Planning for Federal Sustainability in the Next Decade. https://www.gpo.gov/ fdsys/pkg/FR-2015-03-25/pdf/2015-07016.pdf.

[27] Renewable Energy Policy Network for the 21st century (REN21),. 2018. "Renewables 2018-Global status report". https://bit.ly/2Lfjrkv.

[28] I. C. Report. 1973. Dynamic Models for Steam and Hydro Turbines in Power System Studies. IEEE Transactions on Power Apparatus and Systems PAS-92, 6 (Nov 1973), 1904-1915. https://doi.org/10.1109/TPAS.1973.293570

[29] Thierry Van Cutsem and Costas Vournas. 2007. Voltage stability of electric power systems. Springer Science \& Business Media.

[30] V.A. Vinaya Mohanan, I. Mareels, R.J. Evans, and R.R. Kolluri. 2020. The Stabilising Influence of a Synchronous Condenser in Low Inertia Networks. IET Generation Transmission and Distribution (2020). (Accepted).

[31] V.A. Vinaya Mohanan, I. Mareels, R.J. Evans, A.B. Morton, and R.R. Kolluri. 2020. Stability and Bifurcations in Low Inertia PV Rich Power Networks. IET Generation Transmission and Distribution (2020). (Submitted, Under review).

[32] B. Yang, W. Li, Y. Zhao, and X. He. 2010. Design and Analysis of a Grid-Connected Photovoltaic Power System. IEEE Transactions on Power Electronics 25, 4 (April 2010), 992-1000. https://doi.org/10.1109/TPEL.2009.2036432

[33] A. Yazdani, A. R. Di Fazio, H. Ghoddami, M. Russo, M. Kazerani, J. Jatskevich, K. Strunz, S. Leva, and J. A. Martinez. 2011. Modeling Guidelines and a Benchmark for Power System Simulation Studies of Three-Phase Single-Stage Photovoltaic Systems. IEEE Transactions on Power Delivery 26, 2 (April 2011), 1247-1264. https://doi.org/10.1109/TPWRD.2010.2084599

[34] A. Yazdani and R. Iravani. 2010. Voltage-sourced converters in power systems: modeling, control, and applications. John Wiley \& Sons.

[35] O. Zhong, G. C. Konstantopoulos, B. Ren, and M. Krstic. 2018. Improved Synchronverters with Bounded Frequency and Voltage for Smart Grid Integration. IEEE Transactions on Smart Grid 9, 2 (March 2018), 786-796. https: //doi.org/10.1109/TSG.2016.2565663

[36] O. Zhong, P. Nguyen, Z. Ma, and W. Sheng. 2014. Self-Synchronized Synchronverters: Inverters Without a Dedicated Synchronization Unit. IEEE Transactions on Power Electronics 29, 2 (Feb 2014), 617-630. https://doi.org/10.1109/TPEL. 2013.2258684

[37] Q. Zhong and G. Weiss. 2011. Synchronverters: Inverters That Mimic Synchronous Generators. IEEE Transactions on Industrial Electronics 58, 4 (April 2011), 1259-1267. https://doi.org/10.1109/TIE.2010.2048839 